\title{
CoConES: An Approach for Components and Contracts in Embedded Systems
}

\author{
Yolande Berbers, Peter Rigole, Yves Vandewoude, and Stefan Van Baelen \\ DistriNet, Department of Computer Science, KULeuven \\ Celestijnenlaan 200A, B-3001 Heverlee \\ \{Yolande.Berbers, Peter.Rigole, Yves.Vandewoude, Stefan.VanBaelen\} \\ @Cs.kuleuven.ac.be
}

\begin{abstract}
This chapter presents CoConES (Components and Contracts for Embedded Software), a methodology for the development of embedded software, supported by a tool chain. The methodology is based on the composition of reusable components with the addition of a contract principle for modeling nonfunctional constraints. Non-functional constraints are an important aspect of embedded systems, and need to be modeled explicitly. The tool chain contains CCOM, a tool used for the design phase of software development, coupled with DRACO, a middleware layer that supports the component-based architecture at run-time.
\end{abstract}

\section{Introduction}

Embedded systems are typically characterized by a specific functionality in a specific domain, where the software element is taking an increasingly important role. When developing embedded software, besides a range of software quality and stability aspects, one has to consider non-functional aspects and resource constraints. Embedded systems often have limited processing power, storage capacity and network bandwidth. A developer has to cope with these constraints and make sure that the software will be able to run on the constrained system. Often, embedded systems also have timing constraints on their computations. Today, embedded software is becoming complex; according to [8] the complexity of embedded-system applications is increasing with $140 \%$ a year. It is no longer feasible to build such systems from scratch. Reuse of existing software is becoming vital, especially in the light of today's tight time-to-market demands in industry. Reuse should ensure that one can use validated software, only then will reuse result in shorter development time. To enable reuse, we have chosen a component-based approach for building embedded systems.

Component software is quite common today in traditional applications. A large software system often consists of multiple interacting components. These components can be seen as large objects with a clear and well-defined task. Different definitions of a component exist; some see objects as components, while others define components as large parts of coherent code, intended to be reusable and highly documented. We base our definition on the one given by Szyperski [16], see section 2.1. However, many definitions focus only on the functional aspect of a component. For embedded software 
the non-functional constraints cannot be discarded. Modeling these non-functional constraints explicitly enables one to safely reuse components in a design, while being sure that the non-functional constraints will be met. This is the major motivation for the work presented in this chapter.

In the past few years, we have developed a methodology CoCONES (Components and Contracts for Embedded Software) for developing software for embedded systems, using a component oriented approach. Our approach uses contracts to model the nonfunctional constraints. The COCONES methodology is backed by a tool chain that spans both the design-time and the runtime phase. CCOM (Component and Contract-Oriented Modeling) is a software design tool, enabling the developer to specify components and their interactions, including contracts for the non-functional constraints. DRACO (Distrinet Reliable and Adaptive COmponents) is a middleware layer that at runtime will support the component-oriented software architecture on which our methodology is based. It allows components to be created and destroyed, organizes the communication between components, and monitors the contracts defined at design time. Currently, COCONES offers support for memory and bandwidth contracts.

The COCONES methodology is aimed at computationally powerful systems running complex software. Although we address resource-constrained systems, we do not claim to support hard RT applications, nor embedded systems with small footprints.

To validate the various elements of our approach, we have applied our methodology using our tools to various smaller examples and to a fully fledged embedded case study.

This chapter gives a comprehensive overview of our methodology, our componentoriented software architecture, the supporting tool chain and the case study. Elements of this work have been published on conferences and workshops: $[10,11,17,18]$. The presented work was started during the SEESCOA project (Software Engineering for Embedded Systems, using a Component Oriented Approach), and is continued in the CoDAMoS project (Context Driven Adaptation of Mobile Services).

This chapter is organized as follows: Section 2 gives an overview of our component architecture. Sections 3 and 4 respectively describe the design-time tool and the runtime tool that together support our methodology. Section 5 presents a validation of our methodology, component architecture and our tools, through a fully fledged embedded case study. We compare our work with related work in Sec. 6, and conclude in Sec. 7.

\section{Core Concepts of the Proposed Component Architecture}

This section describes the software architecture used in the Components and Contracts for Embedded Software methodology. Before giving details about CoCONES, we list the main strengths and characteristics of COCONES:

1. CoConES components are loosely coupled to facilitate reuse

2. CoCONES components communicate through ports

3. Connectors are used to connect communicating ports

4. CoCONES defines constructs for composing applications out of components

(a) Some constructs describe design-time compositions (blueprints)

(b) Other constructs describe run-time compositions (instances) 
5. Contracts are used to specify and verify non-functional constraints

(a) Contracts can be used to specify and verify non-functional aspects of compositions at design-time

(b) Contracts can be used to verify the correct execution of compositions with regard to their resource use at run-time

(c) Currently, CoCONES supports contracts for timing and for bandwidth requirements

6. CoCONES is a methodology, supported by a CASE tool and by a runtime environment. These tools are described in Sec. 3 and 4.

\subsection{CoCONES Components}

The most common definition of a component was given by Szyperski in [16]: A software component is a unit of composition with contractually specified interfaces and explicit context dependencies only. A software component can be deployed independently and is subject to composition by third parties.

In COCONES, a distinction is made between components and component blueprints. The latter are reusable static entities that only exist at design time and contain a complete description of the type of a component and its implementation (the code). In addition, component blueprints have a unique identifier, a version number and can be stored in a blueprint catalog. In contrast, the term component is reserved for a runtime component instance containing a certain runtime state.

A CoCONES component complies with the definition of Szyperski: it is a reusable documented software entity, offering a coherent behavior and is used as a building block in applications. In addition, all inter-component communication is explicit and takes place by sending asynchronous messages through external interfaces. In general, interfaces are an abstraction of the behavior of a component and consist of a (subset of) interactions of that component, together with a set of constraints on when these interactions may occur. In CoCONES, a component interface consists of a group of messages that may be sent to or sent out from the component. These interfaces are formally specified using the port construct.

\subsection{COCONES Ports}

A CoCONES port represents a bidirectional communication access point of a component, consisting of an interface for incoming messages and an interface for outgoing messages. As with components, the distinction is made between port blueprints and ports. In COCONES, a port is specified on 3 levels:

Syntactic Level: syntactic description of messages that can be sent and received.

Semantic Level: pre and postconditions associated to the messages.

Synchronization Level: description of the sequence in which the messages have to occur.

At the moment of writing, only the syntactic and the synchronization levels have been formally worked out in detail. Two ports can only be interconnected if their associated interfaces match on all levels. The number of connections that can be made with 
a port is specified using the MNOI (Maximum Number of Instances) property of a port. A major advantage of this restriction is that with this additional knowledge about the usage of the component, the developer can make more accurate QoS statements about the services the component delivers. Evidently, these restrictions are enforced at runtime by our execution environment (see Sec. 4). CoCONES supports 3 types of ports with respect to this MNOI:

Single Port: A single port allows for one-on-one communications. This port is represented by a rectangle in our CCOM design tool (Fig. 1(a)).

Multiport: One multiport of dimension $n$ is conceptually identical to $n$ single ports as it allows for $n$ connectors to be attached simultaneously. Although messages can be sent to the entire multiport as such (in this case it behaves as a multicast port), the intended behavior of a multiport is to send messages to a specific index. Conceptually, a multiport is analogous to a call center: a connection is granted to a multiport unless it is already involved in its specified maximum number of connections. Once connected, conversation is one-to-one. As depicted in Fig. 1(b), the symbol of a multiport depends on its dimension.

Multicast Port: A multicast port of dimension $n$ is a single port that can have $n$ connectors attached to it. Messages sent to a multicast port are always sent to all connectors attached to it. It is therefore not possible to differentiate between different receivers. Also, a multicast port can never receive messages. The graphical notation of a multicast port is a trapezium (Fig. 1(c)).

The dimension for both multiports and multicast ports may be $\infty$.

\subsection{CoConES Connectors}

Ports are connected using the connector construct. Compatibility of the port interfaces is checked both at design time and at runtime by the CoCONES tool chain. As such, connectors act as a kind of tunnel during message transmission. Connectors provide a layer of abstraction of component location since they can cross node boundaries when different components are spread over various nodes in a distributed system: components are unaware whether they are communicating with local or with remote components. At runtime, the underlying middleware system (see Sec. 4) takes care of this transparency.

\subsection{CoConES Contracts}

Contracts are used in COCONES to specify non-functional constraints. They allow a designer to impose constraints on the behavior of components and on the interactions between them. Contracts are attached by the designer when an application is constructed by composing components. They can be attached to all previously described constructs of the COCONES architecture.

A COCONES contract is used both for annotation and for verification. It is an important aspect for a designer for documenting applications. Furthermore, contracts are used to verify the correctness of a program regarding their resource use. Some verifications can be done statically and are performed by CCOM, our component composition tool. 


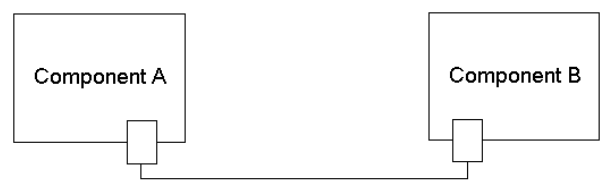

(a) Single Port

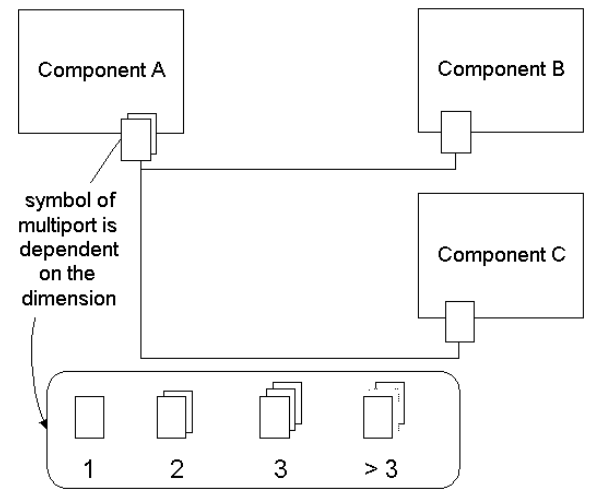

(b) Multi Port

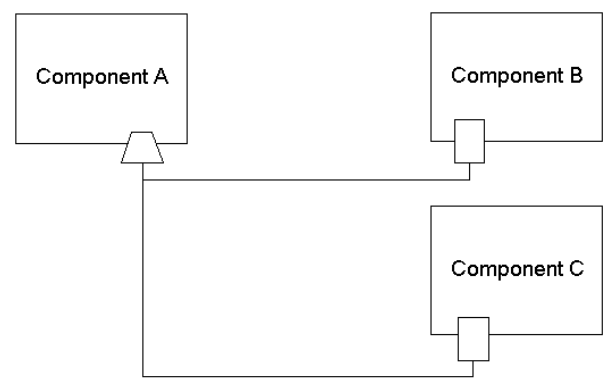

(c) Multicast Port

Fig. 1. Different ports in CoCONES

Other verifications are done dynamically by a contract monitoring module in DRACO (see Sec. 4). Although the CoCONES contracts are a general construct, only timing contracts and bandwidth contracts have been worked out at the time of writing. Work is underway in order to support memory contracts as well.

A COCONES timing contract specifies and imposes the timing constraints to which communicating components have to adhere. Timing contracts can be attached both to connectors and to ports (to specify constraints concerning multiple connections - e.g. $500 \mathrm{~ms}$ after the arrival of message $m$ on port $p 1$, a response must be broad-casted on port $p 2$ ). Two types of timing contracts are currently supported: deadline timing contracts and periodicity timing contracts. A deadline timing contract imposes a constraint on the occurrence time of a particular event, given the occurrence time of an event that happened earlier. Possible events include the sending of a message, the receipt of a message, and the termination of the processing of a received message. A periodicity timing contract imposes a constraint on the periodic occurrence of a particular event. 
CoCONES bandwidth contracts specify constraints concerning the flux density of the information exchanged between two ports. By expressing characteristics of the amount of information exchanged per time unit, we can deduct how suitable a connector is in a distributed component configuration. Therefore, these bandwidth contracts improve the self-containedness of components with regard to their use in a distributed system, making components location transparent. By performing a design-time analysis step that checks the feasibility of the component's connectors over a given connection, the CCOM tool can reject or accept distributed configurations. In order to make bandwidth contracts easily understandable by application engineers using the CCOM tool, CoCONES bandwidth contracts are expressed in terms of concepts that are easy to reason about. Descriptions such as bits per second, available time frames, packets per time unit, etc. make no sense from a component's point of view. Components send out messages at a certain rate, so quantitative aspects of their port's communication behavior should be described in terms of message size (MS) and interval time (IT) between consecutive messages. COCONES bandwidth contracts consist of constraints on several statistical characteristics derived from their message sizes and interval times. Figure 2 gives a conceptual illustration of the relationship between them and the bandwidth they use.

Message Size (MS): the size of a message expressed in bytes.

Interval Time (IT): the time between the beginning of the transmission of two consecutive messages.

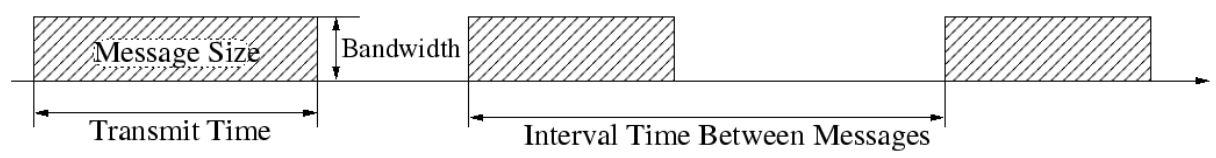

Fig. 2. Message timing

\subsection{CoConES Compositions}

Applications are constructed by creating component compositions. In this process, guided by the CCOM design tool (see Sec. 3), existing component blueprints can be loaded from a component repository and visually be connected to each other. Additional components can be created and key properties (such as the number of ports and their interfaces) can be created by the tool. The CCOM tool generates the necessary skeleton code that can be filled in by the developer. This process is bidirectional, in that the properties of an existing code can be retrieved from its source code. During the design of the application, the CCOM tool will check architectural consistency such as the compatibility of the connected ports on the syntactic and synchronization level, and, where possible, the feasibility of contracts.

\subsection{Supporting Tools}

The entire methodology is supported by a tool chain. The CCOM (Component and Contract-Oriented Modeling) composition tool is a CASE design tool supporting the design and implementation of components and the construction of compositions. CCOM 
is capable of generating skeleton code that assists the developer in the implementation process. The code is then converted to standard Java with a preprocessor, and compiled. At runtime, DRACO (DistriNet Reliable And Adaptive Components) is the middleware system responsible for the correct execution of COCONES compositions. We discuss CCOM and its code generation in Sec. 3. DRACO is discussed in Sec. 4.

\section{CCOM Case Tool}

The CCOM tool supports the development of applications using the architectural CoCONES concepts described in the previous section. First, CCOM assists the developer during the creation and development of:

Component Blueprints: component blueprints can be graphically created, specified and stored into a repository for later use.

Compositions: compositions can be constructed using components (either custom made or retrieved from a repository), connectors and contracts.

In addition, the CCOM tool provides three views in order to decompose the structure of an application. These views allow the developer to focus on the issue at hand, and make sure that relevant constructs are easily accessible:

Blueprint models: all component blueprints from which instances will be used in the composition are grouped in a blueprint model.

Instance models: the instance model gives a structural overview of the application. It consists of connected component instances.

Scenario models: a scenario model represents a specific action in the application. Focus is on non-functional constraints which are represented by contracts that can be attached to component instances, port instances and/or connectors.

The following paragraphs elaborate on the different features of the CCOM tool, and how these features assist the developer in the construction of an application using the CoConES methodology.

\subsection{Developing Component Blueprints}

The development of a component blueprint comprises two steps.

1. The specification of the blueprints of both the component and its ports.

2. Providing an appropriate implementation of the messages a component can receive. This is achieved by filling in the skeleton code that was generated by the CCOM tool during the previous step. Once a component has been specified and implemented, it is transformed into an XML representation and stored in the component repository.

Fig. 3 shows a screen shot of the tool during the development of a blueprint model. In our tool, blueprints are represented with dashed lines. Large rectangles are components, small ones are the ports attached to a component. Left in the figure is the repository of component blueprints, ordered hierarchically. On the right, a blueprint model 


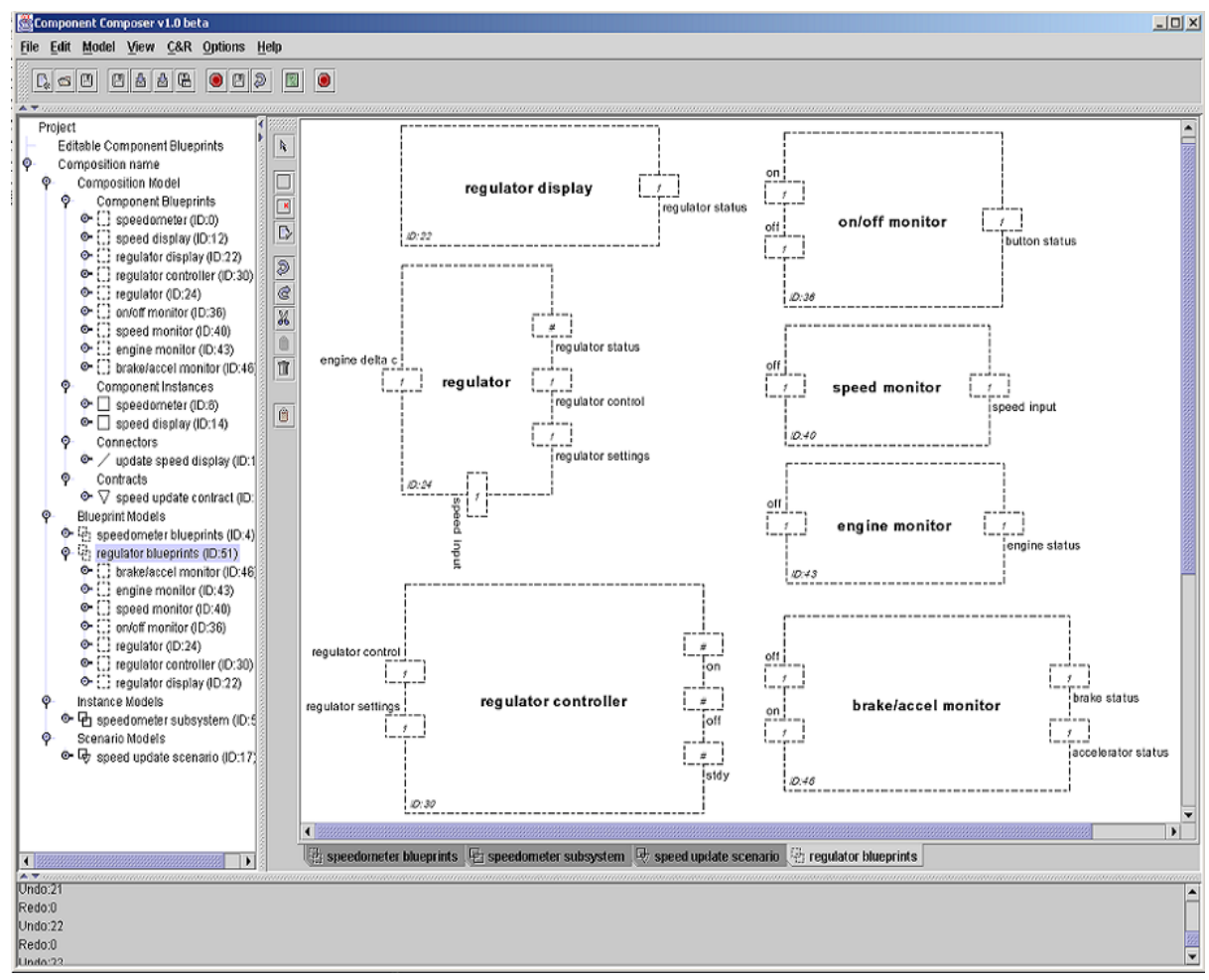

Fig. 3. A blueprint model in the CCOM tool

is shown that groups several component blueprint needed in a car regulator or cruise control application. The cruise control application is used to align the speed of a car to a target speed requested by the driver using a cruise control. The regulator makes use of a speedometer to read the vehicle speed. At a frequency of $2 \mathrm{Hertz}$, that is every $500 \mathrm{~ms}$, the regulator should calculate the new speed of the car, and pass this on to the engine. The regulator should be stopped a.o. when the brakes are hit, the driver accelerates, the driver turns down the cruise control or when the speed drops below a certain limit.

As discussed in Sec. 2.2, port interfaces are specified on multiple levels: syntactic, semantic (not yet implemented) and the synchronization level. The specification of these interfaces is shown in Fig. 4. The port in this figure is the SpeedUpdate port, which is part of the SpeedoMeter component. This component measures the speed of the car at a frequency of 2 Hertz. It can output its speed calculation to an unlimited number of other components. Amongst other, the speed is sent to the Input port of the SpeedDisplay component, which displays the speed on the dashboard of the car.

Fig. 4(a) shows how the syntactic elements of the port blueprint can be filled in: every message can be described including its name, parameters and direction. The synchronization level is shown in Fig. 4(b). Here extended MSC's (Message Sequence Charts) are being used to specify the interaction protocol. From the MSC it becomes clear that the Speedupdate port of the Speedometer component first receives the 
Start message and that it sends Update messages in a loop. The interaction stops when the port receives a Stop message. For each message interaction three hook types can be distinguished (see Fig. 4(b)):

Send hooks (boxes with 'S') representing the sending of messages.

Receive hooks (boxes with ' $\mathbf{R}$ ') representing the reception of messages.

End-of-activation hooks (boxes with ' $X$ ') representing the termination of the processing of a received messages.

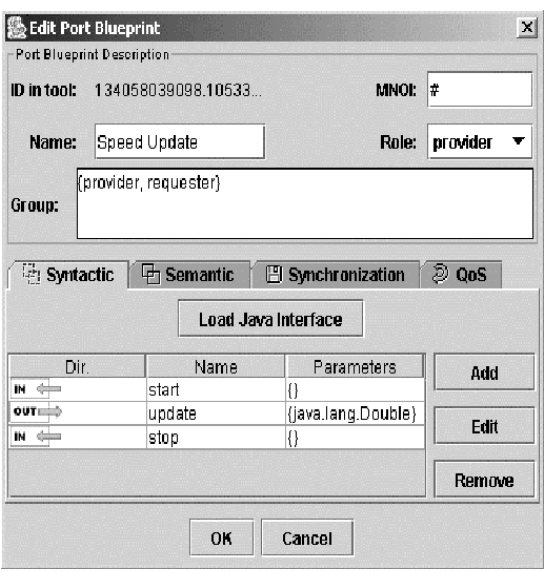

(a) Specification of the Syntactic Interface

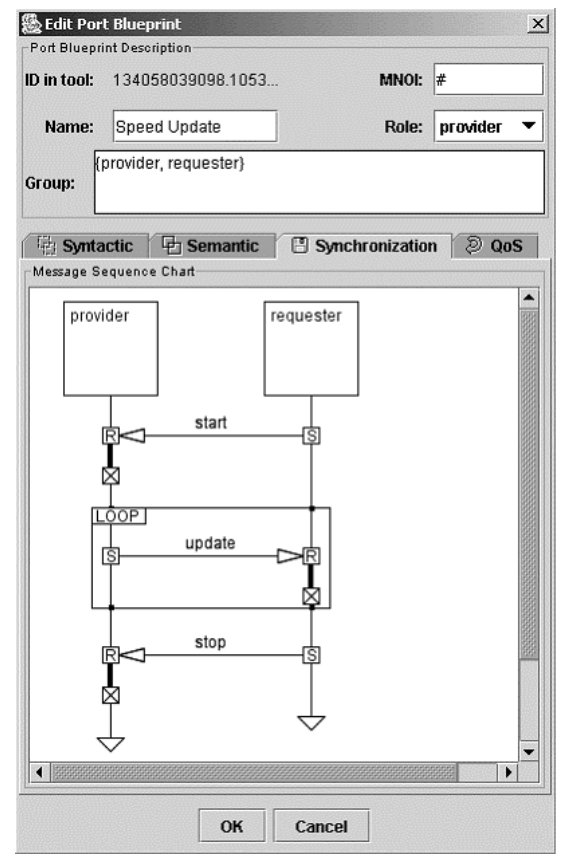

(b) Specification of the Synchronization Interface

Fig. 4. Specification of the interfaces of a port blueprint

The interface of a port blueprint is used to verify whether it can be connected to other port blueprints: connecting ports is only possible if their interfaces match. The compatibility of ports can be verified both for the syntactic level and for the synchronization level. Using the specifications of a component and its ports, the CCOM generates the necessary skeleton code to be filled in by the developer (see Sec. 3.3). The synchronization between a component blueprint specification and its implementation occurs automatically by the CCOM tool.

\subsection{Developing Compositions}

A composition can be built by retrieving component blueprints from the repository and loading them into the composition. Next, instantiations of these component blueprints 
can be created and put into instance models. Connecting component instances is done by (1) instantiating the port instances that will communicate with each other and (2) creating a connector and attaching it to the created port instances.

The scenario model, used in the following step, enables the software developer to impose non-functional constraints on parts of a composition by attaching contracts. A CCOM contract can be attached to one or more participants (component instances, port instances and/or connectors). The actual number and type of participants in a contract are dependent on the particular type of contract: a contract constraining the memory usage of a component is attached to a component instance, while contracts imposing timing constraints on the interaction between components are attached to the ports involved in the interaction.

To make it more concrete, we elaborate here on the timing constraints. In CCOM, timing constraints are specified by means of templates with properties that have to be filled in by the application designer. Using templates makes it easier for a developer to specify constraints, without the need to learn a particular formal specification notation. In general, a CCOM timing contract specifies and imposes the timing constraints to which communicating components have to adhere. A timing contract is concerned with the communication between components. As such, it is straightforward to attach the timing contract to their ports, since these are the communication gateways between components. Furthermore, the communication between components is fully specified by the MSC of the involved ports. So this MSC plays a key role in the specification of a timing contract. A hook is a point on an MSC that represents a particular communication action: we distinguish a send hook, a receive hook and an end-of-activation (eoa) hook. A timing contract can be specified by means of these hooks.

For example, a deadline contract could specify that the maximum duration between the send hook and the eoa hook may not exceed 500 milliseconds. A deadline contract has thus 3 parameters: a hook that starts the contract, a hook that ends the contract, and the maximum allowed time difference between the occurrences of these hooks. The second type of timing contract that CCOM supports is the periodicity timing contract. Fig. 5 shows how such a contract can be specified in our tool. The window in the tool shows the MSC, with the hooks in each message, and the message names. The four necessary parameters of the periodicity contract can be filled in at the bottom: in this example the contract starts at the sending of the start message, the periodic event is the reception of the update message, the contract ends with the sending of the stop message, and the period is $500 \mathrm{~ms}$.

\subsection{From Design to Execution: Code Generation}

To facilitate the development of components in CoCONES, the skeleton code of the component and its ports is generated by the CCOM tool. CCOM also ensures synchronization between a component blueprint specification and its implementation. For the further implementation of the component, a custom language is used. This language is a superset of Java that supports relevant component-based constructs. The code is automatically preprocessed, compiled and packaged into a binary that is ready for deployment in the runtime environment. As such, the design is directly used as input for the implementation. 


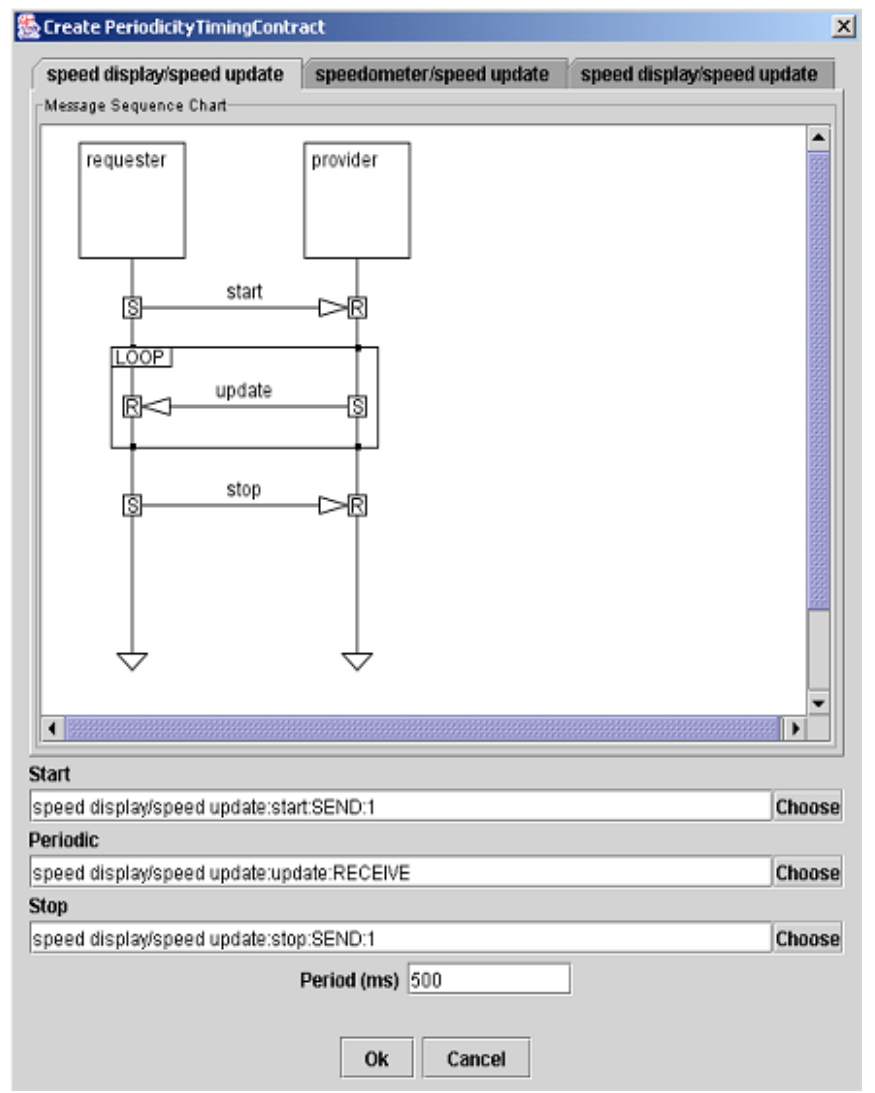

Fig. 5. The specification of a periodicity timing contract in the CCOM tool

We shortly illustrate the language with a small example consisting of two components that are used in our cruise control example: a speedoMeter and a speed Display. The first component will measure the speed of a vehicle using an existing method (measurespeed ( ) ) and will broadcast this information on its Out port. The second component prints out all values it receives through its Input port. The implementation of these two components is shown in Fig. 6.

The implementation of a component starts with the component keyword. It consists of zero or more attributes (e.g. \$activated in Fig. 6) and methods (not shown for these trivial components) and the description of its ports. The declaration of a multicast port is straightforward since it can not accept messages. It suffices to specify its existence using the multicastport keyword. Two parameters are required: the name of the port and the maximum number of simultaneous connections that are allowed. In the above example, the multicastport Out specifies an UNLIMITED number of simultaneous connections. As such, connections will never be refused at runtime. A multiport has a similar declaration, but since it can accept messages, these messages must be declared as well using the message keyword. The definition of a message in- 


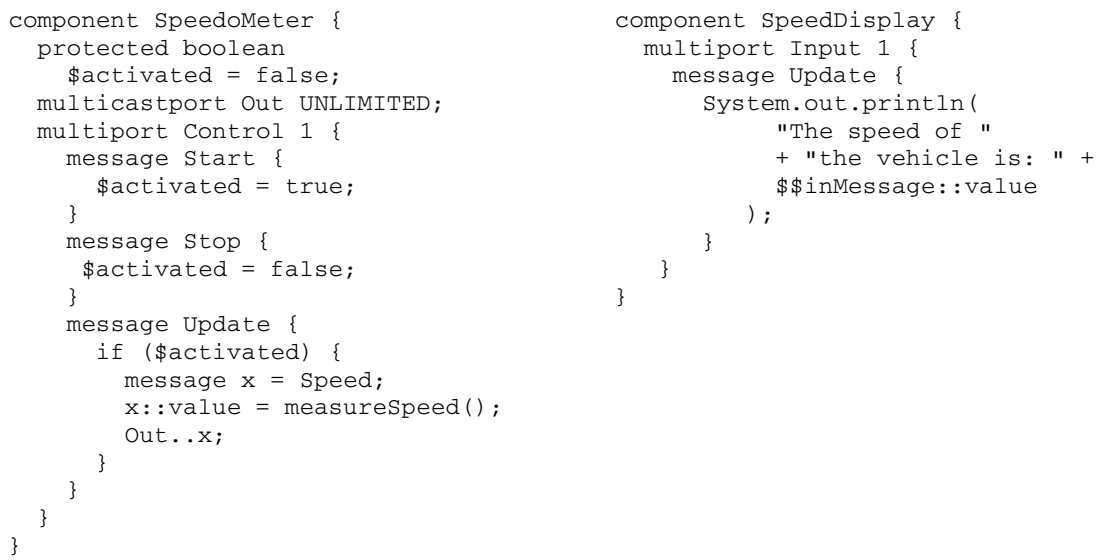

Fig. 6. Two simple components in CoConES notation

cludes the code to be executed when the message is received on the port. New messages can be created using the statement:

$$
\text { message varName = messageName; }
$$

After its creation, this message can be sent out through any connected port. Message sending is asynchronous and as such, the sending of a message is always successful. If the component on the other side of the connector does not accept messageName, the system will return a CannotDeliverMessage message. Finally, two additional operators were added. Fields of a message are accessed using the : : operator:

$$
x: \text { : value = measureSpeed }() ;
$$

The . . operator is used on a port to send out a given message:

$$
\text { Out . .x; }
$$

Inside the implementation body for a message, the implicit parameter $\$$ \$inMessage refers to the received message. In the Update message of the SpeedDisplay component for instance (see Fig. 6), a parameter is retrieved from the incoming message and displayed on screen.

\section{Draco Runtime System}

Next to the CCOM tool, the tool chain supporting the CoCONES methodology also contains a runtime environment capable of executing CoCONES compositions: the DRACO component system. DRACO is a middleware system that provides the underlying infrastructure of an execution environment for component compositions. The runtime system is highly modularized. As such, it can be configured and targeted to specific applications, while guaranteeing a minimal memory footprint. The DRACO system is implemented in Java and targeted towards more powerful embedded systems such as an IPAQ or a robot used in manufacturing. Very small embedded systems or systems with 
hard real-time deadlines, such as often found in the automotive world are not the focus of the DRACO middleware platform. The CoConES component design-methodology can however, be used on such systems as well.

The architecture of DRACO is depicted in Fig. 7. It consists of a core system which provides the minimal functionality to execute CoConES applications. The most important tasks of the core system are as follows:

1. Management of component instances, connectors and contracts.

2. Support for introspection and naming.

3. Abstraction of the underlying hardware and OS.

4. Routing and scheduling messages sent between components.

The core system consists of 5 units and its footprint is less than $65 \mathrm{kB}$, allowing it to be installed on embedded devices with stringent resource constraints. At startup, the core is dynamically assembled using the builder pattern [7]. Since the builder reads an XML file describing which implementation to use for each of the core units, modifying or replacing one core unit has no impact whatsoever on the rest of the system. The ability to easily customize its core makes DRACO an excellent platform for various assessments (e.g. replacing the scheduler would allow us to investigate the influence of the scheduling algorithm on the execution of a component based application, ...). Furthermore, it allows for further customization depending on the target platform. Once instantiated, the core is considered to be fixed. In order to keep the complexity (and size) of DRACO sufficiently low, no attempt was made to allow for unanticipated modifications of the DRACO core at runtime. The 5 core modules are:

Component Manager: is responsible for loading component blueprints, creating instances and removing them. It also keeps a repository of created component instances, with a basic directory mechanism mapping names onto component instances.

Connector Manager: is a repository containing the connectors that exist between component instances in a composition. Each connector refers to the ports to which it is connected. Each port has a send message handler queue and a receive message handler queue associated to it.

Message Manager: this module is responsible for delivering messages sent out by components. By means of the Connector Manager it retrieves the send message handler queue of the sending port and the receive message handler queue of the receiving port. The messages then traverse the send message handler queue of the sending port and arrive at the Scheduler.

Scheduler: accepts messages coming from a send message handler queue and schedules them for delivery to the appropriate message handler queue.

Module Manager: responsible for loading and unloading extension modules, which can be used to extend the functionality of the DRACO component system.

As shown on top of Fig. 7, each of the core modules exports a lightweight component-interface. These appear as components in the runtime system, and can be used by application components to query or configure the underlying middleware environment. 


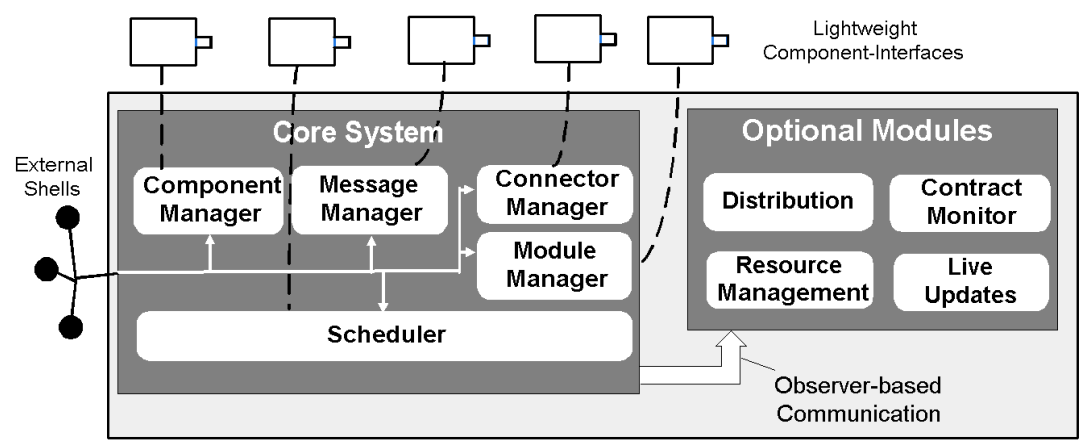

Fig. 7. Overview of the DRACO architecture

Interaction between DRACO and the user is handled by an external shell, which is provided to DRACO at startup and resides in a different binary. By separating the user interaction from DRACO, it is possible to use different interaction shells depending on the situation. An interactive shell with scripting capabilities is available for use during development on a high performance desktop machine, while a thin layer with minimal functionality can be used when resource consumption is an issue.

Although the functionality of the core system is relatively limited, DRACO offers an infrastructure which allows the addition of functionality that may not always be required: extension modules. These modules can be loaded and unloaded at runtime by the module manager. The following extension modules have been worked out:

The distribution module (DM): this module adds distribution functionality to the core platform in a complete transparent way. It introduces the notion of proxy components, similar to the proxy pattern defined in [7]. These proxy components are lightweight components that represent remote components. As such, they offer the same ports and exactly the same semantic information. The DM is responsible for (1) setting up and tearing down connections between remote DRACO systems, and (2) responsible for managing proxy components and generating them based on real components. In DRACO, a connection is an abstract concept that can be implemented by any kind of physical wired or wireless connection. No stubs or other design-time entities need to be generated in advance in order to make components communicate in a distributed way. Instead, proxies are created dynamically on an as-needed basis. This incorporates a considerable advantage over traditional approaches that need additional constructs (e.g. the subs an skeletons used by Java RMI).

The contract monitor: this module checks whether contracts are violated at runtime. Depending on the type of the contract, the monitoring differs. For the timing contracts, messages are intercepted and time stamped by an event gathering unit. These time stamps are used by the event processing unit to verify the timing contract. The unit responsible for the verification of the contract, can be moved to another node to minimize intrusion on the target platform. Currently, contract violations are reported offline: a developer can analyze the occurred violations after an application's execution. In the future, a contract violation will be reported to the application, which then must take corrective measures. 
The resource manager: this extension module is responsible for the negotiation of contracts with applications when these are started. The resource manager knows what contracts are currently active, and can then accept new contracts in function of available resources.

The live update module (LUM): allows components to be replaced at runtime, even while they are part of a running application. The LUM achieves this by (1) putting the component to be replaced in an inactive state by temporarily holding back the messages, (2) instantiating the new version of the component, (3) possibly transferring the internal state from the old components to the new components, using routines provided by the developers of the components, (4) rewiring the connectors of the old version to the new component, (5) activating the new component by deblocking the messages that were held back in step 1 (6) removing the old component.

Since the exact tasks and thus requirements of extension modules are unknown in advance, they can make use of reflection mechanisms and may subscribe to one of the many events triggered by the core system.

In addition, they can interfere with the message flow and interact with the delivery of messages. In DRACO, messages are sent asynchronously between components. The path followed by a message traveling from component A to component B consists of 3 major parts (see Fig. 8(a)): the sending message chain, the scheduler and the receiving message chain. Each extension module can add message handlers to these message chains to implement the features they want.

The sending message chain comprises the journey of a message from the moment it is sent through the port of the originating component until it is scheduled for execution by the scheduler. Its detailed implementation in DRACO is shown in Fig. 8(b).

In the first step, the component contacts the port through which the message will be sent. Since ports are implemented as inner classes in DRACO, this is achieved with a local call (arrow 1). The port will pass on the message to the message manager (arrow 2) which will retrieve the attached connector from the connector manager (arrow 3). Each connector is associated with 4 message handlers (the first handler of both the sending and receiving chain of each direction: component $A$ to $B$ and vice versa). The message manager retrieves the two handlers associated with the current message direction. The receiving message handler is used for the delivery of the message after it has been scheduled for execution by the scheduler (see further). It is therefore simply passed on with the message to the sending message handler (arrow 4). This sending message handler is the first (and in the most basic scenario also the last) handler in a chain of message handlers. Each handler in the chain has the ability to intercept and modify the message, and will then forward it to the next handler in the chain (arrow(s) 5). The last handler is responsible for the delivery to the scheduler (arrow 6).

After receiving both the message and its associated receiving message handler, the scheduler queues the message until it is ready for execution. The exact queuing mechanism depends on the scheduler that is used, but it is the responsibility of the scheduler to preserve the order of messages over a given connector.

When the scheduler has selected a message for delivery, it allocates a thread for the execution of this message, and passes on the message to its receiving message handler. 


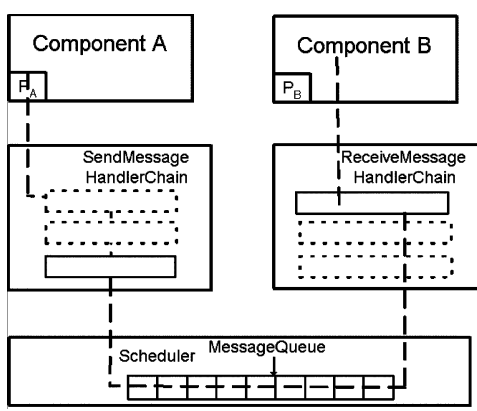

(a) Schematic overview of message journey

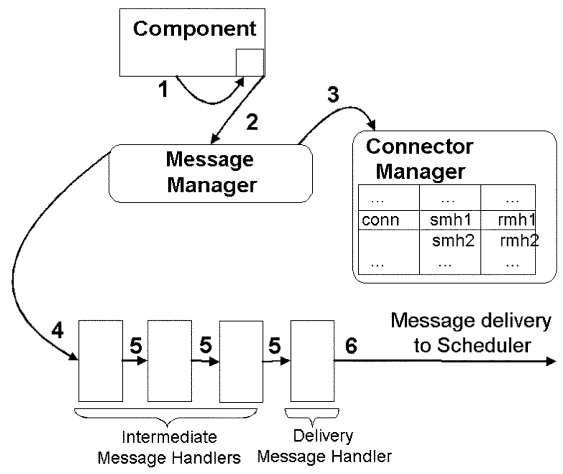

(b) Details of the sending message chain

Fig. 8. Message Delivery in DRACO

As shown in Fig. 8(a), the principle behind the receiving sequence is identical to the sending sequence: there is a chain of message handlers that process the message (e.g. the timing monitor can read out the time stamp added to the message by his peer in the sending message chain) and subsequently pass it on to the next handler in the chain. The last handler delivers the message to the port at the end of the connector. This port will then dispatch the message to the actual method associated to the message. After message execution, control is returned to the scheduler.

\section{Extensive Case Study}

Several embedded applications have been developed using the CoCoNES design methodology and the supporting tool chain. One of these is the car regulator introduced in Sec. 3. This application has been used as a case study to define and assess timing constraints. However, as our implementation was not run on a car, and not on embedded hardware, it was in our eyes still a toy application.

We then developed a full fledged embedded application, with the specific intention to validate our design methodology in a larger application, to verify the specific advantages of our component architecture and to test the re-usability of our components and our designs. This led to a camera surveillance system of which several variations were designed and implemented.

The surveillance system can be used for security related purposes such as physical intrusion detection and registration of activity in home and office buildings. A PC/104 embedded computer (holding the operating system, a Java virtual machine, our component runtime and the test case code) was connected to a DFW-VL500 firewire digital camera. It was linked over a TCP/IP network with a desktop PC serving as a storage and control station.

The two bottom boxes in Fig. 9 (generated by the CCOM tool) give an overview of the component compositions in the surveillance case. Device boundaries are indicated by surrounding boxes. The central Camera component on the embedded device (PC 


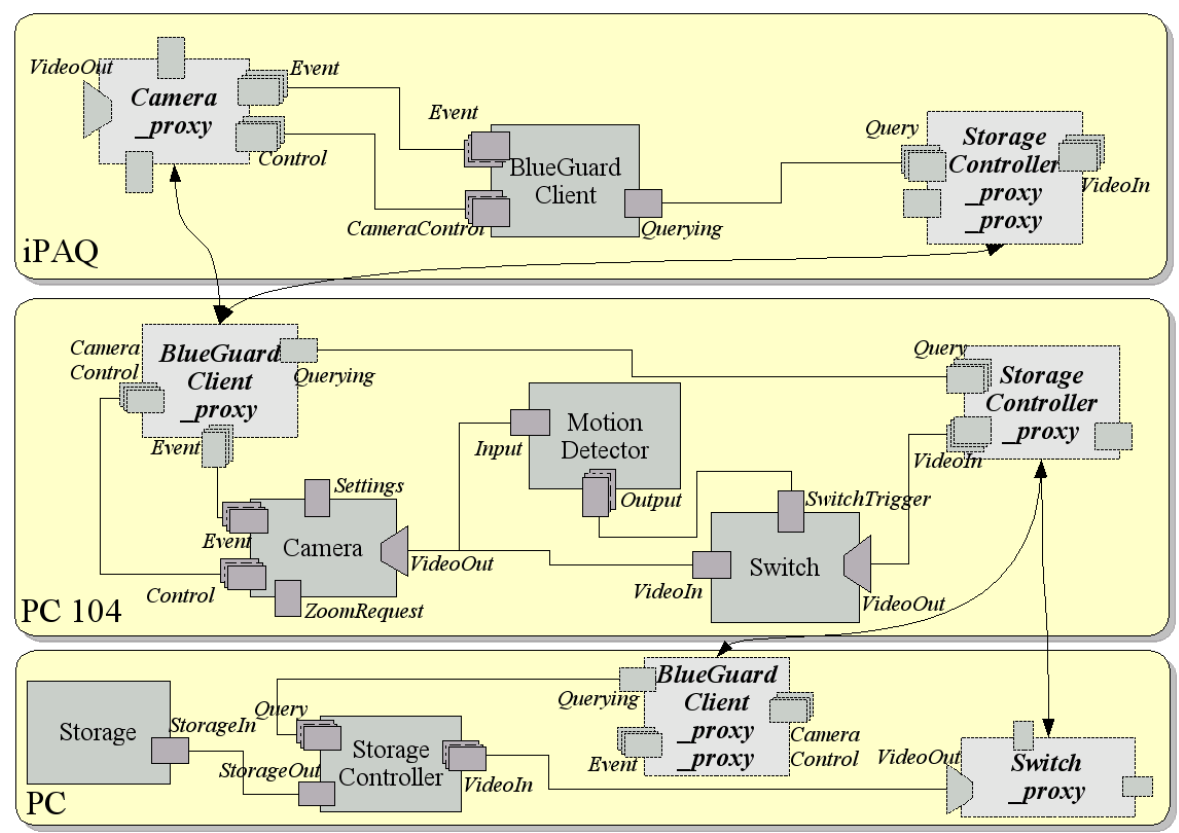

Fig. 9. Overview of the Camera Surveillance Case

104) continuously grabs images from the camera at a predefined rate and multicasts them towards the MotionDetector and the Switch component. The motion detector analyzes the images and produces an alarm-start output message when motion is detected. The switch, receiving the message from the motion detector, forwards the video stream towards its output port until the alarm-stop message is received, meaning that motion has ceased. The suspicious images are sent to the StorageController component, which is located on the desktop PC. Proxy components are introduced for handling the remote communication transparently. The Storagecontroller. Proxy and the Swi tch_Proxy allow for remote communication between Storage Controller and Switch. The Storage component, which encapsulates database access, eventually stores the images. The core application, as just described, was developed in CCOM and executes on top of DRACO, running a distribution module.

In order to demonstrate reuse, several variations and extensions of the core application were developed. In one of the variations the motion detector component and the switch component were replaced by a component that passes one image out of 20 to the storage controller. This was a straight forward change, and all the other components could be reused without any change.

Subsequently, we wanted to take reuse one step further and ported our component system on an iPAQ. We then designed BlueGuard which provides security guards with more information about the safety inside the building. This extension allows the guards to query the recorded images using a handheld device when they are in the neighborhood of an observation station. For this extension a BlueGuardClient component was created that is available on the handheld device. 


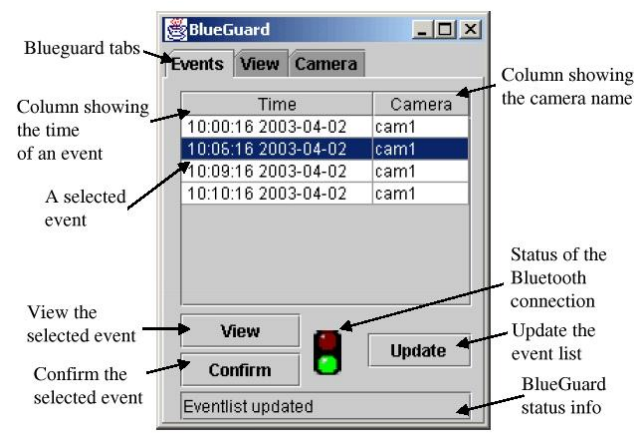

(a) Viewing events

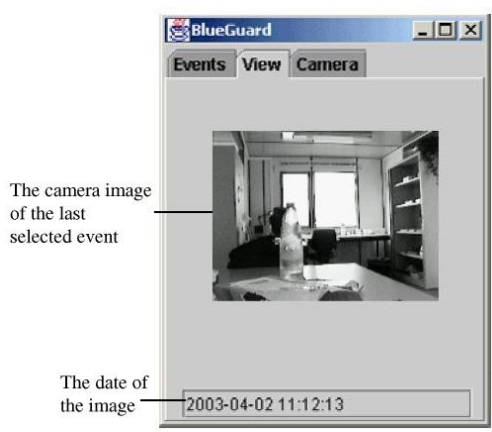

(b) Viewing an image

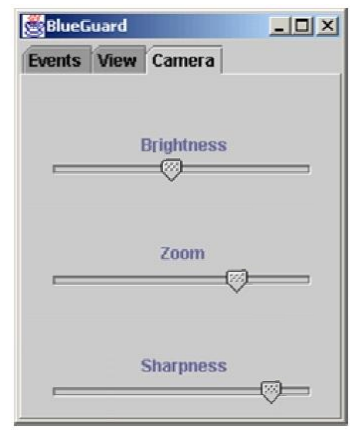

(c) Camera Control

Fig. 10. BlueGuard client

Furthermore, the distribution module was extended with Bluetooth [3] connection support to enable short-range wireless access to our observation stations. The Blue GuardClient component, instantiated on the iPAQ handheld devices (see the top box of Fig. 9), provides the user interface used by the security guards. This component can be connected to a StorageController component for querying purposes and to several Camera components for adjusting camera settings such as focus and zoom. As depicted on the example setup (Fig. 9), the BlueGuardClient may be connected to a StorageController component through the embedded PC 104 module using proxy components for routing their messages. Fig. 10(a) shows the tab view of all recent events and the Bluetooth connection status. The image associated with each event can be requested and is shown in the view tab (Fig. 10(b)). The third tab (Fig. 10(c)) allows for changing the brightness, sharpness and zoom parameters of the camera. An in-depth description of this BlueGuard extension can be found in [11].

Both timing contracts and bandwidth contracts have proven their advantages in the surveillance case. In the distributed setup, the bandwidth contract attached to the connection between the Switch and StorageController component imposed limitations on the rate at which the camera's images could be sent. At the resolution of $320 \times 240$ in uncompressed RGB color format, we were limited to sending no more than 4 images per second over a $10 \mathrm{Mb}$ Ethernet connection. The message size 
is 230400 bytes and the interval time 250 milliseconds. The Bluetooth communication between the handheld device and PC 104 module was fast enough for transmitting requested images to the handheld device because of the long interval times between consecutive images (only one image at the time is viewed). However, the timing contract describing the time between sending the image and receiving it only just met the timing requirements due to the rather slow $(0.4 \mathrm{Mbps})$ throughput. The periodicity contract attached to the image sending hook of the camera was defined with a period of 250 milliseconds, fitting the requirements imposed by the network connection. Contract monitoring proved that they were always adhered by the components during the test period.

Our case study allowed us also to validate the live update possibilities of our architecture. To do so, we have replaced the MotionDetector component by a newer version, using a different algorithm, while the application was running. Both the old and the new version compare every image with the previous one. The state of this component consists of the current image. It was this state that was asked to the old component and fed to the new component. The application as a whole continued to work fine. No images were lost.

The core camera surveillance system, its variations and its extensions, have proven the soundness of our methodology and our approach where components and contracts play a central role, have shown that our architecture is suitable for embedded platforms, and have validated our tool chain.

\section{Relation to the State-of-the-Art}

In [2], Beugnard et al. argue that component interfaces should be specified on 4 levels: basic, behavioral, synchronization and Quality of Service. This is similar to the specification of port interfaces in CoCONES. The last level of the port specification was deliberately omitted, since Quality of Service properties are specified using contracts. CoCONES contracts are more general and have a broader scope. Therefore, they can be attached to components or connectors as well (e.g. memory contracts are likely to be attached to components), and are not tied exclusively to a port.

The state-of-the-art of component based development is too large to be presented here. We just contrast our work with alternative component-based frameworks specifically targeted to embedded systems, which have been developed in recent years.

In Koala [19], components are implemented in $\mathrm{C}$ and specify provides and requires interfaces that cannot be changed. Interfaces can be connected if the provided interface implements at least all methods for the required interface. The binding of these interfaces is made at the product level. All external information (including memory management) must be retrieved through require interfaces.

Other embedded component systems worth mentioning are PECOS [9, 20] (a model for field-devices with the emphasis on formal execution models using Petri Nets), PortBased Objects [14, 15] (used in the Chimera RT operating system), VEST (A tool set for constructing and analyzing component based embedded systems) [13] and DESS (a generic component architecture and notation for embedded software development) $[5,6]$. 
CoConES has several aspects in common with the Ptolemy II project [1]. Ptolemy II studies the modeling, simulation and design of concurrent, real-time, embedded systems. It specifically focuses on the assembly of concurrent components and the use of models of computation that regulates the interaction between embedded components. One of their major problem areas are the use of heterogeneous mixtures of models of computation, including discrete-event systems, data flow, process networks, synchronous and reactive systems, and communicating sequential processes.

A number of COCONES concepts are inspired by ROOM [12] and UML-RT, more specifically components, ports, and connectors. Although initially intended for designing and building telecommunication systems, the ROOM methodology can also be used for the design of other types of embedded systems. ROOM designs contain primarily actors, ports, bindings and state machines. The ROOM methodology has some new and interesting ideas: it introduces thread encapsulation which hides the internal thread mechanisms; it offers and alternative way of connecting software components by means of bindings; the idea of port protocols is an advantage since it enforces a designer to only connect compatible ports; and it offers executable models and the ability to generate code by putting code into transitions of the state machines. ROOM however lacks a consistent way to annotate time in designs. In general, ROOM has no support for the annotation of non-functional constraints, like for instance memory and bandwidth constraints.

The Fractal component model [4] in particular is very interesting because it is in several aspects based on the same principles as CoCONES's component model. Support for extension and adaptation is its prime concern and it is aiming at a broad range of host devices from embedded systems to application servers. It has, however, a less strictly defined component definition than COCONES's components and provides a language independent interface definition for its components. This interface definition can be used to connect components written in different languages. In addition, there is also support for composite components and it allows (sub)components to be shared between components. In the Fractal model, connections between two or several components are called bindings. There are two types of bindings, primitive bindings and composite bindings. Primitive bindings are language-level bindings (synchronous or asynchronous) whereas composite bindings are a composition of primitive bindings and components. Using this definition of inter-component bindings, flexible distributed applications can be built. Each component has a component controller that can control all internal behavior of the component such as affecting operation invocations, influencing the behavior of internal components, creating new components, etc.. The Javabased Fractal framework that supports the Fractal component model consists of a core and several extensions, called increments. Like in DRACO, these increments can add new functionality to the core. The core offers a basic API for performing actions such as creating components, adding bindings between components and managing the content of components. Some of the increments under development allow for component bootstrapping, component distribution (distributed bindings), mobility and protection (resource management and distribution). 


\section{Conclusion}

This chapter has given an overview of CoCONES, a methodology and architecture for developing software for embedded systems, using a component oriented approach, where contracts are used to model the non-functional constraints. CoCONES is backed by a tool chain that spans both the design-time and the runtime phase. Contracts can be specified at design-time and can be checked both at design-time and at runtime. The runtime environment offers beside its core system support for distribution and live updates. A full fledged embedded case study has proven the soundness of our methodology, the applicability of our software architecture in the domain of embedded systems and the robustness of our runtime environment.

In conclusion we can say that our methodology is original in that it is supported by a tool chain, where both functional and non-functional constraints are checked, and this both at design-time and at runtime. These checks are generated by the tools, based on the design made by the developer. The key contribution of our work is therefore the integrated tool chain that spans design-time and runtime, and covers functional and non-functional constraints. More detailed description of this work can be found in [10, $17,18]$.

CoConES is an ongoing project. We are currently on different tracks to extend and improve our methodology and architecture. (1) We are extending the contracting framework to support general resource contracts in an extensible way. This framework will make it easy to add new types of contracts to the tool chain and monitoring mechanisms for new contracts to the DRACO runtime system. One track that is already being explored is the deployment of memory contracts and mechanisms to monitor a component's memory use. In addition, the new contract framework will allow resource contracts to be negotiable at deployment time and even renegotiable when resource availability in the system changes. This will enable flexible runtime deployment of programs on embedded systems without endangering the system's robustness and reliability. (2) In the future, our live updating tool will be able to automatically generate state transfer functions in order to enhance the support for updating components at runtime. (3) With the addition of the notion of context, our future runtime system will be able to discover, retrieve and process context information to improve its application's capabilities to react to environmental conditions and events. Such context information could include information about the user of the system (such as his preferences), environmental information (such as temperature, location and time), platform information (such as CPU and memory information) and service information (available software services). (4) Furthermore, research is being conducted on runtime software adaptation and reconfiguration mechanisms that respect existing resource contracts. This way, programs can -guided by the runtime system- adjust its configuration and composition at runtime according to new contextual conditions.

\section{Acknowledgment}

Both projects introduced, SEESCOA and CoDAMoS, are funded by the Belgian Institute for the Promotion of Innovation by Science and Technology in Flanders. 


\section{References}

1. Philip Baldwin, Sanjeev Kohli, Edward A. Lee, Xiaojun Liu, and Yang Zhao. Modeling of sensor nets in ptolemy ii. In Proceedings of Information Processing in Sensor Networks (IPSN), Berkeley, CA, USA, April 26-27 2004.

2. Antoine Beugnard, Jean-Marc Jézéquel, Noël Plouzeau, and Damien Watkins. Making components contract aware. Computer, 32(7):38-45, July 1999.

3. Bluetooth. Bluetooth wireless protocol, 2003. http://www.bluetooth.com/ and http://www.bluetooth.org/.

4. Eric Bruneton, Thierry Coupaye, and Jean-Bernard Stefani. Recursive and dynamic software composition with sharing. In Proc. of the Seventh International Workshop on ComponentOriented Programming, Malaga, Spain, 2002.

5. DESSteam. Definition of components and notation for components. Technical report, December 2001. http://www.dess-itea.org.

6. DESSteam. Timing, memory and other resource constraints. Technical report, 2001. http://www.dess-itea.org.

7. Erich Gamma, Richard Helm, Ralph Johnson, and John Vlissides. Design Patterns: Elements of Reusable Object-Oriented Software. Addison Wesley, 1994.

8. ITRS. International technology roadmap for semiconductors. Internet, 2004. http://public.itrs.net.

9. Oscar Nierstrasz, Gabriela Ar'evalo, Stéphane Ducasse, Roel Wuyts, Peter Müller, C. Zeidler, Thomas Genssler, and R. van den Born. A component model for field devices. In In proceedings of the IFIP/ACM working conference on Component Deployment, Berlin, 2002.

10. Peter Rigole, Yolande Berbers, and Tom Holvoet. Design and run-time bandwidth contracts for pervasive computing middleware. In C. Urarahy, A. Sztajnberg, and R. Cerqueira, editors, Proceedings of the first International Workshop on Middleware for Pervasive and Ad Hoc Computing (MPAC)., pages 5-12, Rio De Janeiro, Brazil, June 2003.

11. Peter Rigole, Yolande Berbers, and Tom Holvoet. Bluetooth enabled interaction in a distributed camera surveillance system. In Proceedings of the Thirty-Seventh Annual Hawaii International Conference on System Sciences, pages 1-10. IEEE Computer Society, 2004.

12. B. Selic, G. Gullekson, and P. Ward. Real-Time Object Oriented Modeling. Wiley, 1994. ISBN 0471599174.

13. John A. Stankovic. VEST - A toolset for constructing and analyzing component based embedded systems. Lecture Notes in Computer Science, 2211:390-402, 2001.

14. David B. Stewart and Pradeep K. Khosla. The chimera methodology: Designing dynamically reconfigurable and reusable real-time software using port-based objects. International Journal of Software Engineering and Knowledge Engineering, 6(2):249-277, June 1996.

15. David B. Stewart, Richard A. Volpe, and Pradeep K. Khosla. Design of dynamically reconfigurable real-time software using port-based objects. Software Engineering, 23(12):759-776, 1997.

16. Clemens Szyperski. Component Software: Beyond Object-Oriented Programming. AddisonWesley, November 2002.

17. David Urting, Stefan Van Baelen, Tom Holvoet, Peter Rigole, Yves Vandewoude, and Yolande Berbers. A tool for component based design of embedded software. In J. Noble and J. Potter, editors, Proceedings of 40th International Conference on Technology of Object-Oriented Languages and Systems (Tools Pacific 2002), volume 10, pages 159-168, Sydney, Australia, February 2002. Australian Computer Society Inc.

18. David Urting, Tom Holvoet, and Yolande Berbers. Embedded software development: Components and contracts. In T. Gonzalez, editor, Proc. of the IASTED Conference on Parallel and Distributed Computing and Systems, pages 685-690, 2001. 
19. Rob van Ommering. Building Reliable Component-Based Software Systems, chapter The Koala Component Model. Artech House Publishers, July 2002.

20. Michael Winter, Thomas Genssler, Alexander Christoph, Oscar Nierstrasz, Stéphane Ducasse, Roel Wuyts, Gabriela Arévalo, Peter Müller, Chris Stich, and Bastiaan Schönhage. Components for embedded software - the pecos approach. In Proceedings of the Second International Workshop on Composition Languages, Malaga, Spain, June 2002. 